\title{
Delayed Osteon Formation in Long-Bone Diaphysis of an 11-Year-Old Giant Cow with Dermal Dysplasia
}

\author{
Ryoichi MORI, Tetsuo KODAKA ${ }^{1)}$ and Yoshihisa NAITO ${ }^{2)}$ \\ First and ${ }^{1)}$ Second Departments of Oral Anatomy, Showa University School of Dentistry, Tokyo 142-8555, ${ }^{21}$ Department of Veterinary \\ Internal Medicine, Faculty of Agriculture, Iwate University, Iwate 020-8550, Japan
}

(Received 2 June 1998/Accepted 1 October 1998)

ABSTRACT. The transverse sections of radius diaphysis in an 11-year-old giant Holstein cow with dermal dysplasia of a collagen disorderrelated skin fragility (Cow 1), probably based on increasing turnover of the dermal collagen as reported previously, were morphologically and physico-chemically investigated. Cow 1 had about one and a half times as much as the body weight of normal Holstein cows, aged 5 to 6.5 years with stabilized growth. The bone samples were compared with those of a 12-year-old Holstein cow as controls (Cow 2). It has been reported that the long-bone diaphysis of young calves and some herbivorous dinosaurs are occupied with laminar bone showing a concentric appositional formation, and that such a laminar bone is characteristically seen during the growing period of some farm animals and large dogs that show very rapid growth rates. Cow 1 had a smaller number of osteons than Cow 2 in the outer-half layer of the diaphysis, and showed an intermediate type between Cow 2 and a 1-year-old Holstein ox in the entire layers, although their bone volumes were similar among them. There were no significant differences in $\mathrm{Ca}$ and $\mathrm{P}$ concentrations and the Vickers microhardness values between the bone matrix of Cow 1 and Cow 2. The bone-collagen fibrils of Cow 1 showed uneven diameters and a disordered arrangement. Thus, there may be some relation in collagen formation between the bone matrix of Cow 1 and the dermis. From the remaining volume of laminar bone, Cow 1, aged 11 years, had probably shown growth until quite recently, so that we consider that Cow 1 became a giant animal, in the same way as some herbivorous dinosaurs.-KEY wORDs: compact bone, delayed osteon formation, giant bovine, histology, laminar bone.

J. Vet. Med. Sci. 61(2): 101-106, 1999

It has been reported that the compact substance of longbone diaphysis in young calves shows a wire-netting bone [1], a primary plexiform tissue [6], or a laminar bone [2, 7, 11]. In this study, we used the term laminar bone. The tissue in the transverse sections of long-bone diaphysis basically shows an alternative concentric structure of the bone lamellae and the cavities of blood vessels around the bone-marrow periphery $[1-3,6,7,11]$, and one unit of laminar bone contains a bright line [2] or a hypercalcified primear in the center [3]. The laminar bone, which has been formed without bone-remodeling for a certain period of time after birth, is gradually replaced by the so-called Haversian bone with numerous osteons followed by aging $[2,7]$. In addition, such a laminar bone is characteristically seen during the growing period of some farm animals and large dogs that show very rapid growth rates [11]. However, the appearance time and succeeding period of osteons in calves and other animals have not been elucidated yet.

Recently, we had an opportunity to observe the compact bone of radius diaphysis in a giant Holstein cow. The cow, when aged 2 years, had been found to have dermal dysplasia of a collagen disorder-related skin fragility, probably based on increasing turnover of the dermal collagen by biochemical investigations [8]. In clinical observations, the dermal dysplasia continued until the cow was sacrificed at 11 years after birth [10]. In the 2-year-old cow, the dermis showed an anomalous deposition of thin collagen fibers with van Gieson staining, and the collagen fibrils had ragged outlines in cross sections by transmission electron microscopy [8].
In this study, for the purpose of studying the structure of bone matrix and also for comparing with the dermal collagen fibrils $[8,10]$, we investigated the radius diaphysis of the giant cow by using microradiography, backscattered electron (BSE) imaging, image analyzing, electron probe microanalyses including wave-dispersive X-ray (WDX) and energy-dispersive X-ray (EDX), microhardness test, and scanning electron microscopy. As controls, a 12-year-old Holstein cow and a 1-year-old Holstein ox were employed.

\section{MATERIALS AND METHODS}

Animals: The 11-year-old Holstein cow with dermal dysplasia of a collagen disorder-related skin fragility [8] was sacrificed, and the radius was removed. The cow (Cow 1) showed a heavier body weight (about $1,000 \mathrm{~kg}$ ) [10] than normal Holstein cows (about $650 \mathrm{~kg}$ ), aged 5 to 6.5 years, thus showing a maximum level in growth [9]. In clinical observations, Cow 1 was found to be in good health except for the dermal dysplasia and her behavior to be normal [11]. For controls, the radius of a 12-year-old normal Holstein cow (Cow 2) and the tibia of a 1-year-old normal Holstein ox (1-year ox) were applied. Their diaphyses were cut transversely into several slices with a diamond wheel and then fixed with $10 \%$ buffered formalin, $70 \%$ ethanol, or a 4\% Karnovsky's fixative solution.

Formalin-fixed samples for morphological observations and the evaluations of hardness values: The radius-diaphysis slices of Cow 1 and Cow 2 were cut transversely again into 3 sections, respectively. In the first pair of Cow 1 and Cow 
2 sections, they were ground with grindstones down to 100 $\mu \mathrm{m}$ in thickness, and contact microradigraphs were taken with a soft X-ray device (SRO-M50; Sofron, Tokyo, Japan).

In the second pair of Cow 1 and Cow 2 sections, and the tibia slice of a 1-year ox, the transverse cut surfaces were polished with 5- and $0.3-\mu \mathrm{m}$ alumina on the polishing clothes. Their polished surfaces were observed after carbon coating by BSE imaging in a scanning electron microscope (SEM: S-2500CX; Hitachi, Tokyo, Japan) operated at an accelerating voltage of $25 \mathrm{kV}$. By using such BSE images, the thickness of the compact bone from the endosteum to the periosteum was equally divided into 5 concentric layers, and then 5 radial zones of 1-mm width from the endosteum to the periosteum were randomly selected for the measurements of bone volume and osteon number. The bone volume in each layer was measured with a personal image analysis system (LA-525; Pias, Osaka, Japan) and the mean ratio of bone volume was calculated. The osteon number in each layer was counted and the mean osteon number $\left(\mathrm{N} / \mathrm{mm}^{2} \times 5\right.$ layers $)$ was calculated.

In the third pair of Cow 1 and Cow 2 sections, the transverse surfaces were polished and the Vickers microhardness values were then measured by using a reflected light microscope with differential interference contrast (Photomicroscope III; Zeiss, Oberkochen, Germany) fitted with a microhardness tester (MHP; Zeiss) under a loading of $50 \mathrm{~g}$ for $15 \mathrm{sec}$. Five Vickers indentations were randomly made in each layer and the mean Vickers microhardness number $(\mathrm{Hv})$ was calculated $(n=50: 10$ points $\times 5$ layers).

Ethanol-fixed samples for the evaluation of calcification values: The transverse cut surfaces of radius-diaphysis slices in Cow 1 and Cow 2 were polished. After carbon coating, the $\mathrm{Ca}$ and $\mathrm{P}$ concentrations in the compact bone from the periosteum to the endosteum were anlyzed with a S-2500CX SEM equipped with a WDX spectrometer (WDX-3PC; Microspec, Fremont, CA, U.S.A.). The microprobe conditions were an accelerating voltage of $25 \mathrm{kV}$ and a specimen irradiation current of $1 \times 10^{-5} \mathrm{~mA}$. The full scale of the Ca concentration was 5,000 count per sec (cps) and that of the $\mathrm{P}$ concentration was 2,000 cps. Quantitatively, $\mathrm{Ca}$ and $\mathrm{P}$ concentrations were analyzed at 20 points in the inner basic lamellae with an S-2500CX SEM equipped with an EDX detection system (Delta-4; Kevex, Foster City, CA, U.S.A.). The microprobe conditions were an accelerating voltage of $15 \mathrm{kV}$, a specimen irradiation current of $1 \times 10^{-7}$ $\mathrm{mA}$, and a beam spot of $15 \mathrm{~nm}$. The counting time was 100 sec and the standard sample was native fluorapatite.

Karnovsky's fixed samples for SEM observations: The radius-diaphysis slices of Cow 1 and Cow 2 were cut longitudinally along the long axis and decalcified with $10 \%$ ethylenediamine tetra-acetic acid at $\mathrm{pH}$ 7.2. After freezing with liquid nitrogen, the samples were fractured transversely. The fractured samples were treated with a $2 \%$ tripsin solution for removal of organic matter except for collagen fibrils, and then they were soaked in a $2 \%$ osmium and a $2 \%$ tannic-acid solution for conductive staining. This was followed by dehydrating with ethanol and critical-point drying with $\mathrm{CO}_{2}$. The specimens were observed with a field-emission type SEM (S-700; Hitachi) operated at an accelerating voltage of $20 \mathrm{kV}$ after coating with an about 10-nm-thick platinum-palladium layer.

\section{RESULTS}

Bone histology: Figure 1 shows contact microradiographs of the transverse ground sections of Cow 1 and Cow 2. In Cow 2, osteons showing relatively dark (low-calcified) and small dotted areae were distributed throughout the entire layers, but there were fewer osteons in Cow 1, especially in the outer-half layer showing a concentric laminated structure. Figure 2 shows BSE images of the outer-third layer in the transverse polished surfaces of Cow 1 and Cow 2. Osteons containing Haversian canals in the center were densely packed in Cow 2, but Cow 1 had only a few osteons and the laminar bone was concentrically arranged. One unit of laminar bone had a hypercalcified primear or a bright line in the center.

Bone volume: Figure 3 shows BSE images of the outerthird layer in the transverse polished surfaces of Cow 1, Cow 2, and a 1-year ox. Cow 1 was mainly formed by laminar bone whereas Cow 2 was occupied with numerous osteons (Fig. 2). A 1-year ox was formed by laminar bone similar to Cow 1, and the hypercalcified primear or bright line was clearer than that of Cow 1. From the BSE images as shown in Fig. 3, the bone volume was calculated in the entire layers from the endosteum to the periosteum (Fig. 4). The bone volumes of Cow 1, Cow 2, and a 1-year ox were $98.3,96.7$, and $97.9 \%$, respectively. Their bone volumes were similar to each other.

Density of osteon number: Figure 5 shows a histograph on the density of the osteon number $\left(\mathrm{N} / \mathrm{mm}^{2} \times 5\right.$ layers $)$

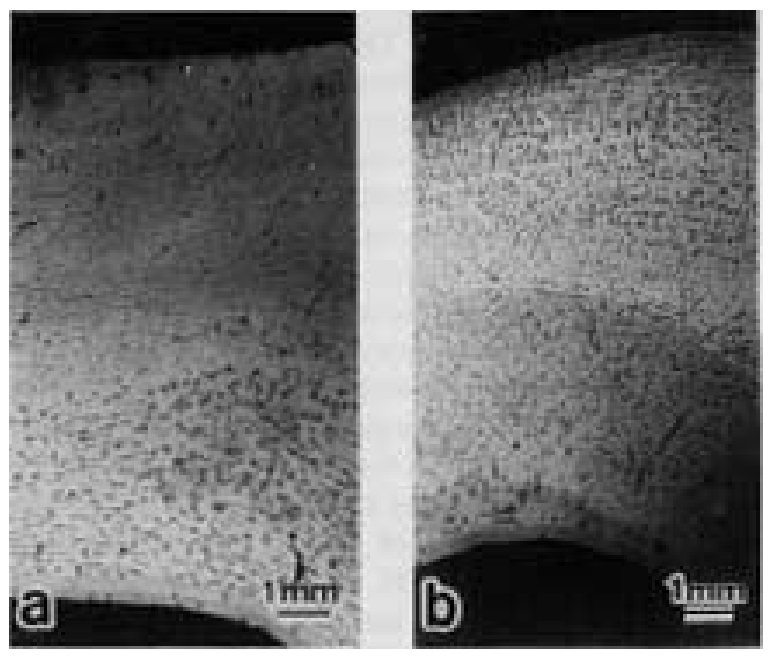

Fig. 1. Microradiographs of the transverse ground sections of radius diaphysis. Cow 1 (a) and Cow 2 (b). Relatively dark and small dotted areae are osteons. Upper side: periosteum. Lower side: endosteum. 

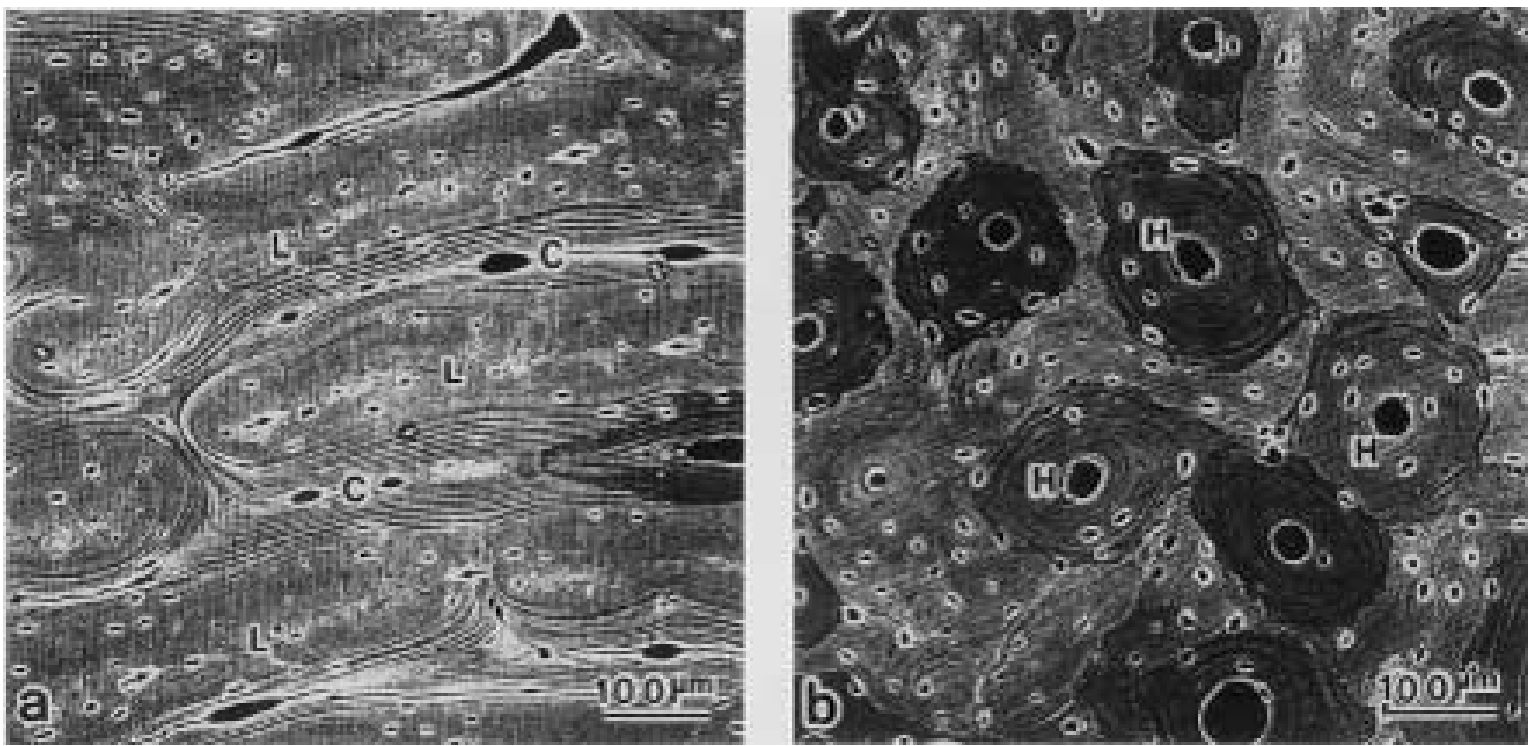

Fig. 2. Backscattered electron (BSE) images of the outer-third layer in the transverse polished surfaces of radius diaphysis. Cow 1 (a) and Cow 2 (b). L: laminar bone. C: cavity of blood vessels. H: Haversian canal of an osteon. Smaller areae showing a low BSE signal are osteocyte lacunae. Arrow: hypercalcified primear.
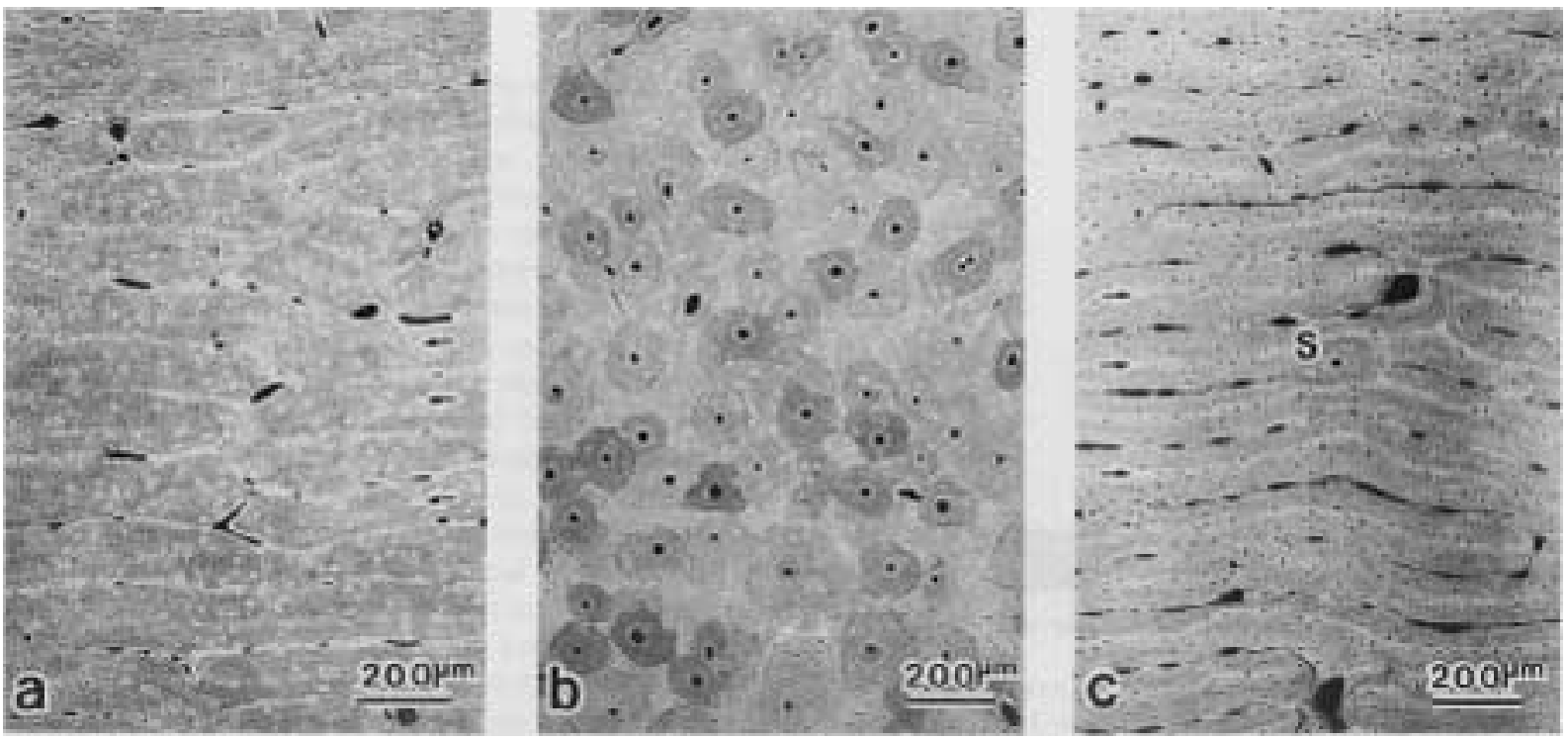

Fig. 3. Backscattered electron (BSE) images of the outer-third layer in the transverse polished surfaces of radius and tibia diaphysis. Cow 1 (a) and Cow 2 (b), a 1-year ox (c). Laminar bone (a, c) and Haversian bone with osteons (b). Arrow: hypercalcified primear. S: pseudosteon.

based on the BSE images as shown in Fig. 3. The osteon number of Cow 2 was about equally distributed in the entire layers, but Cow 1, mainly occupied with laminar bone in the outer-half layer, had a smaller number of osteons in the outer (IV and V) layers, while the inner (I and II) layers were similar to those of Cow 2. The middle (III) layer of Cow 1 formed a successional zone from laminar bone to Haversian bone. In a 1-year ox, the osteon number was small in the inner layers and the outer layers had few osteons. That is, the density of the osteon number in Cow 1 was an intermediate type between that in Cow 2 and a 1-year ox.

Calcification values: Figure 6 shows distribution patterns of $\mathrm{Ca}$ and $\mathrm{P}$ concentrations in the transverse polished surfaces from the periosteum to the endosteum in Cow 1 and Cow 2 by WDX microanalysis. Their distribution patterns of Cow 1 were similar to those of Cow 2. By EDX microanalysis of the inner basic lamellae, statistically, there were no significant $(p<0.01)$ differences in $\mathrm{Ca}$ and $\mathrm{P}$ concentrations, and $\mathrm{Ca} / \mathrm{P}$ molar ratio between Cow 1 and Cow 2 (Table 1). 


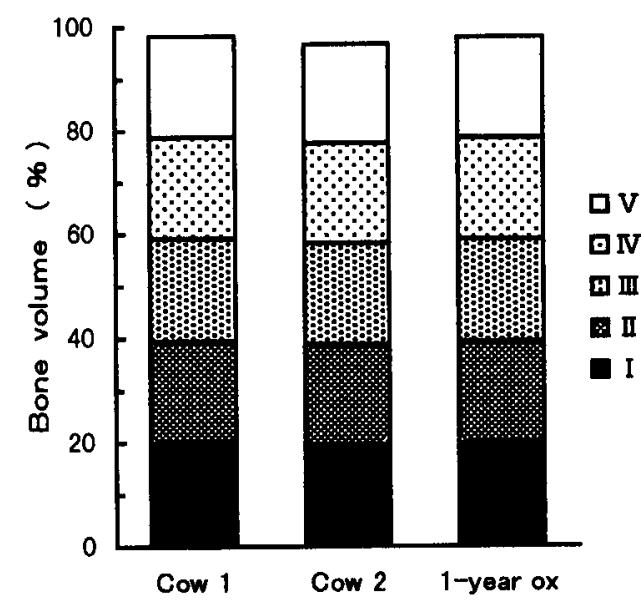

Fig. 4. Bone volumes on the transverse polished surfaces of radius (Cow 1 and Cow 2) and tibia diaphysis (1-year ox). The compact bone from the endosteum to the periosteum is equally divided into 5 concentric layers. The 5 layers of $\mathrm{I}$ to $\mathrm{V}$ indicate the inner $1 / 5$, inner $2 / 5$, middle, outer $2 / 5$, and outer $1 / 5$, respectively. Based on the BSE images as shown in Fig. 3.

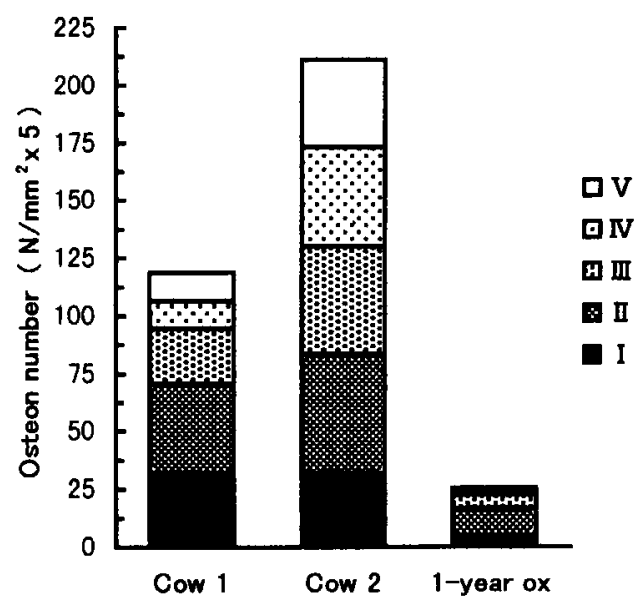

Fig. 5. Osteon numbers on the transverse polished surfaces of radius (Cow 1 and Cow 2) and tibia diaphysis (1-year ox). Unit: osteon number $/ \mathrm{mm}^{2} \times$ 5 layers. The 5 layers of $\mathrm{I}$ to $\mathrm{V}$ are the same as shown in Fig. 4. Based on the BSE images as shown in Fig. 3.

Hardness values: Table 2 shows Vickers microhardness values in the entire layers of the transverse polished surfaces in Cow 1 and Cow 2. Statistically, there was no significant $(p<0.01)$ difference in the hardness values between them.

SEM of bone-collagen fibrils: Collagen volume of Cow 1 was similar to that of Cow 2 with a SEM at low magnifications in the decalcified tissue of radius diaphysis. At higher magnifications, the collagen fibrils in the innerthird layer of Cow 1, where osteons were present, showed uneven diameters and a disordered arrangement compared

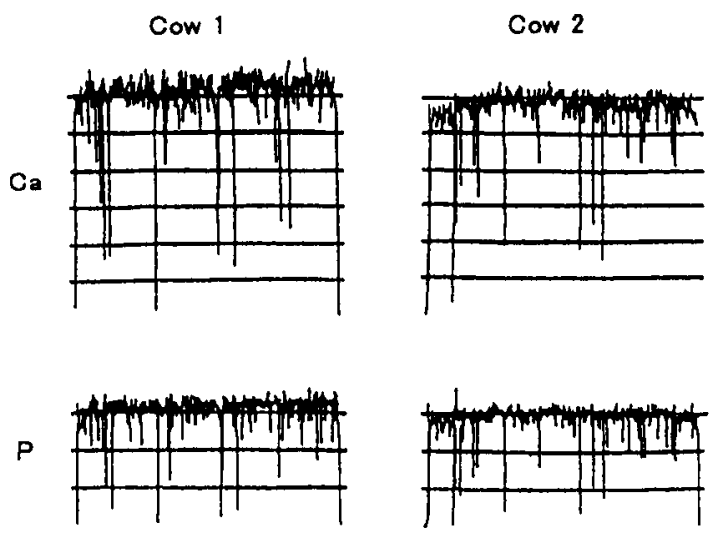

Fig. 6. Distribution patterns of $\mathrm{Ca}$ and $\mathrm{P}$ concentrations in the transverse polished surfaces of radius diaphysis. In each graph, the periosteum is left and the endosteum is right. WDX microanalysis.

with those of Cow 2 (Fig. 7). Similar collagen fibrils were observed in the outer-third layer of Cow 1, which were almost occupied with laminar bone.

\section{DISCUSSION}

Bone histology and osteon number of Cow 1: Laminar bone including such terms as primary plexiform tissue and wire-netting bone, which has been reported in calves, pigs, and other large animals, basically shows an alternative concentric structure of the bone lamellae and the cavities of blood vessels around the periosteum in the transverse planes of the long-bone diaphyses $[1-3,6,7,11]$. One laminarbone unit contains a bright line [2] or a hypercalcified primear in the center [3], surrounded with woven bone and also with lamellar bone adjacent to the cavities of blood vessels in both sides [2]. The laminar bone is formed by the periosteum and the appositional formation may accelerate the growth of long-bone thickness [2]. In addition, such a laminar bone is characteristically seen during the growing period of some farm animals and large dogs that show very rapid growth rates [11]. Followed by aging, osteons appearing in the inner layer of compact bone gradually replace the laminar bone, and then the Haversian bone consisting of osteons is formed [2, 7]. However, the appearance time and succeeding period of osteons in calves and other animals have not been elucidated yet.

There were similar bone volumes in the transverse surfaces of long-bone diaphyses among Cow 1 aged 11 years, Cow 2 aged 12 years, and a 1-year ox, but the density of the osteon number in Cow 1 showed an intermediate type between Cow 2 and a 1-year ox. Thereby, Cow 1 had a larger volume of laminar bone than Cow 2. These data mean that there had been delayed osteon formation in the long bones of Cow 1. From the densities of the osteon numbers of Cow 1 and a 1-year ox in each layer (see Fig. 5), the osteon formation probably starts from the innermost layer adjacent to the bone marrow which contains osteoclasts. 

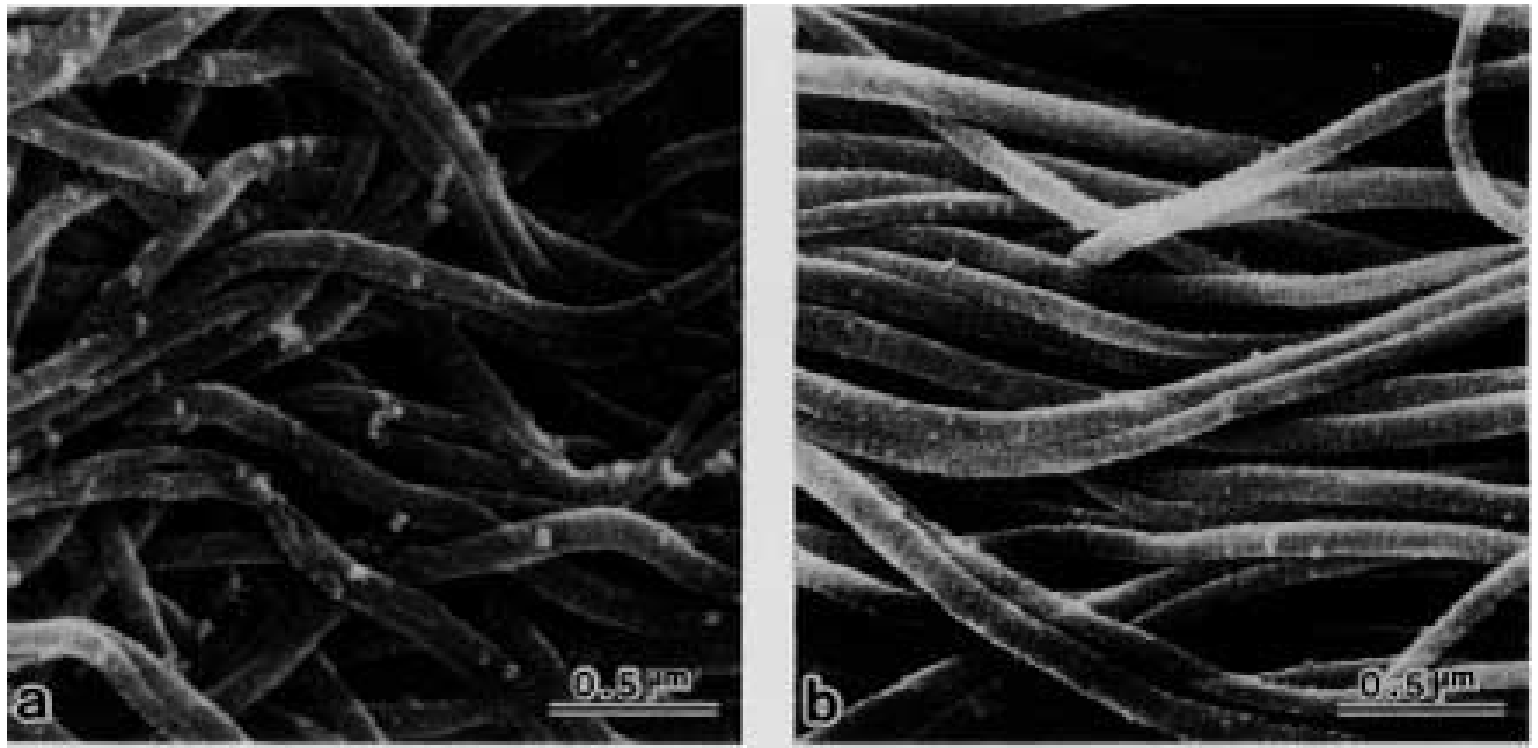

Fig. 7. Scanning electron micrographs of collagen fibrils in the inner-third layer of radius diaphysis. Cow 1 (a) and Cow 2 (b). Decalcified bone samples.

Table 1. Concentrations of $\mathrm{Ca}$ and $\mathrm{P}$, and $\mathrm{Ca} / \mathrm{P}$ molar ratios in the inner basic lamellae of radius diaphysis by EDX microanalysis

\begin{tabular}{llll}
\hline & \multicolumn{1}{c}{$\mathrm{Ca}$} & \multicolumn{1}{c}{$\mathrm{P}$} & \multicolumn{1}{c}{$\mathrm{Ca} / \mathrm{P}$} \\
\hline Cow 1 & $24.04 \pm 0.79^{\mathrm{a})}$ & $10.38 \pm 0.43^{\mathrm{a})}$ & $1.80 \pm 0.02^{\mathrm{b})}$ \\
Cow 2 & $24.01 \pm 0.54$ & $10.47 \pm 0.80$ & $1.78 \pm 0.03$ \\
\hline
\end{tabular}

a) Weight percentage (Mean \pm S.D., $n=20$ ).

b) Molar ratio (Mean \pm S.D., $n=20$ ).

There are no significant $(p<0.01)$ differences in $\mathrm{Ca}$ and $\mathrm{P}$ concentrations, and $\mathrm{Ca} / \mathrm{P}$ molar ratios between Cow 1 and Cow 2 .

The laminar bone has also been reported in some herbivorous dinosaurs possessing heavy body weight $[5,7$, 12]. The body weight of giant Cow 1 , when aged 2 years, showed about $600 \mathrm{~kg}$ [10] which was heavier than the about $500 \mathrm{~kg}$ in similar generation Holstein cows [9]. When aged 11 years, Cow 1 (about 1,000 kg) [10] had about one and a half times as much as the body weight of normal Holstein cows (about $650 \mathrm{~kg}$ ), aged 5 to 6.5 years, with stabilized growth [9]. From the remaining volume of laminar bone $[2,11]$, Cow 1 had probably shown growth until quite recently, so that we consider Cow 1 became a giant animal, in the same way as some herbivorous dinosaurs [5, 7, 12].

Chemical and physical characteristics of Cow 1 bone: From $\mathrm{Ca} / \mathrm{P}$ molar ratios, the bone mineral of Cow 1 was carbonate-containing apatite [4] similar to that of Cow 2. There were no significant differences in $\mathrm{Ca}$ and $\mathrm{P}$ concentrations and the Vickers microhardness values between Cow 1 and Cow 2. Their bone volumes were similar to each other as mentioned already. Currey [2] suggested that laminar bone had a more intimate bloodsupply than Haversian bone and showed that it had a larger
Table 2. Vickers microhardness values in the transverse polished surfaces of radius diaphysis from the endosteum to the periosteum

\begin{tabular}{cc}
\hline & Vickers hardness value \\
\hline Cow 1 & $79.9 \pm 8.0^{\mathrm{a})}$ \\
Cow 2 & $80.8 \pm 7.5$ \\
\hline
\end{tabular}

a) Vickers hardness number: Hv (Mean \pm S.D., $n=50$ ). There is no significant $(p<0.01)$ difference in the $\mathrm{Hv}$ between Cow 1 and Cow 2.

surface area of blood-channel per unit volume by using young and older calves and pigs. These suggestions mean that laminar bone shows a better bone-metabolism than Haversian bone.

The radius of Cow 1 , which retained a relatively large amount of laminar bone until it was sacrificed at 11 years after birth, was in good health except for the dermal dysplasia and her behavior was normal in clinical observations [10]. Therefore, we suggest that Cow 1 should have had no problems in bone metabolism as suggested by Currey [2]. Moreover, the long bones of Cow 1 had been probably strong against physical stress, because the laminar bone showing a concentric wire-netting structure as shown in Figs. 3a and 3c [1, 2], similar to one with usual osteons, may have a mechanical structure as if one giant osteon were present in a long bone.

Comparison of collagen fibrils in Cow 1 bone and the dermal dysplasia: Cow 1 had the dermal dysplasia of a collagen disorder-related skin fragility, probably based on increasing turnover of the dermal collagen [8]. The dermis of Cow 1, when aged 2 years, showed an anomalous deposition of thin collagen fibers with van Gieson staining, and the collagen fibrils had ragged outlines in cross sections 
by transmission electron microscopy [8]. Dhem et al. [3] reported that there was a difference in the amount of bonecollagen fibers between a normal newborn calf and a newborn calf with dermatosparaxis showing a hereditary disorder of the connective tissue.

In the compact bone of Cow 1, however, bone-collagen fibers were not loose with a SEM at low magnifications, whereas the collagen fibrils showed variable diameters and a disordered arrangement at higher magnifications. The latter findings may resemble those in the dermis [8]. In general, calcified bone-collagen fibrils remain there until the bone matrix is absorbed by osteoclasts. In the bone matrix of Cow 1, a larger amount of newly-formed collagen fibrils which might be immediately calcified during laminar bone formation without bone-remodeling [2], would not be able to increase turnover as the dermal-collagen fibrils [8], but might cause long bones to become larger.

ACKNOWLEDGMENTS. We gratefully thank staffs of the Department of Veterinary Internal Medicine, Faculty Agriculture, Iwate University, for their invaluable cooperation. We are also grateful to Prof. R. Takiguchi, First Department of Oral Anatomy, Showa University School of Dentistry, and Dr. T. Kawaguchi, Department of Biochemistry, Iwate Medical College School of Dentistry, Iwate, Japan, who supplied precise support and suggestion. This work was partly supported by a grant-in-aid for Scientific Research from the Ministry of Education in Japan (No. 09670030).

\section{REFERENCES}

1. Brouwer, E. 1952. Over Beenstructure en Voeding bij Mens en Dier. Voeding 13: 55-65.

2. Currey, J. D. 1960. Differences in the blood-supply of bone of different histological types. Quart. J. Microsc. Sci. 101: 351-370.

3. Dhem, A., Piret, N., Nicaise, M. and Nusgens, B. 1976. Bone in dermatosparaxis. I. morphological analysis. Calcif. Tiss. Res. 21: 29-36.

4. Driessens, F. C. M. 1982. Mineral aspects of dentistry. pp. 1215. In: Monographs in Oral Science, vol. 10 (Myers, H. M. ed.), Karger, Basel.

5. Enlow, D. H. and Brown, S. O. 1957. A comparative histological study of fossil and recent bone tissues, part II. Texas J. Sci. 9: 186-214.

6. Enlow, D. H. and Brown, S. O. 1958. A comparative histological study of fossil and recent bone tissues, part III. Texas J. Sci. 10: 187-230.

7. Halstead, L. B. 1974. Vertebrate Hard Tissues. pp. 64-72. Wykeham Pub., London.

8. Kawaguchi, T., Fukazawa, H., Naito, Y. and Okada, K. 1988. Dermal dysplasia characterized by collagen disorder-related skin fragility in a cow. Am. J. Vet. Res. 49: 965-971.

9. Naito, M. 1973. Breeding of daily cattle. pp. 100-127. In: Dictionary of Zootechnical Science (Sasaki, K. ed.), Youkendo, Tokyo (in Japanese).

10. Naito, Y., Kawaguchi, T. and Okada, K. 1994. Dermal dysplasia. J. Clin. Vet. Med. 12: 39-44 (in Japanese).

11. Palmer, N. 1993. Bone and joints. pp. 1-182. In: Pathology of Domestic Animals, 4th edn., vol. 1 (Jubb, K. V. F., Kennedy, P. C. and Palmer, N. eds.), Academic Press, San Diego.

12. Ricqles, A. J. de 1980. Tissue structures of dinosaur bone, functional significance and possible relation to dinosaur physiology. pp. 103-139. In: A Cold Look at the Warm-Blooded Dinosaurs (Thomas, R. D. K. and Oslon, E. C. eds.), Westview Press, Boulder. 